\title{
EDITORIAL
}

\section{Práctica basada en la evidencia}

L

os profesionales de enfermería, en la práctica, se encuentran continuamente con la necesidad de tomar decisiones. Por lo general, las decisiones son basadas en conocimientos adquiridos en su formación; de indicaciones de otros profesionales o de la experiencia propia ${ }^{(1)}$. Sin embargo, la toma de decisiones en el cuidado de las personas se debe apoyar en la utilización de la evidencia científica disponible, es decir, respaldadas por el mejor conocimiento de investigación disponible a partir de revisiones sistemáticas, metaanálisis y meta-síntesis ${ }^{(2)}$.Esto favorece la relación entre la teoría, investigación y práctica, al generar conocimiento para realizar cambios en la prácti$\mathrm{Ca}^{(3)}$. Además una práctica basada en evidencia logra los mejores resultados de cuidado y cumple con las expectativas de las personas, asimismo en las Instituciones de Salud aumenta los indicadores de calidad de la atención ${ }^{(4)}$. En este sentido la Secretaria de Salud, ha promovido la síntesis de la mejor evidencia de investigación en el cuidado por equipos de investigadores expertos y clínicos. Estas síntesis de investigación, han proporcionado la base para desarrollar directrices sólidas basadas en la evidencia para la práctica: guías de práctica clínica. Estas guías identifican el mejor plan de tratamiento o estándar de oro para el cuidado y promueven resultados de salud de calidad ${ }^{(5)}$. Los mayores beneficios de la práctica basada en evidencia son mejores resultados para las personas de cuidado, proveedores e instituciones de salud. El profesional de enfermería tiene fácil acceso a numerosas guías basadas en evidencia para tomar las mejores decisiones clínicas respecto al cuidado.

En la práctica diaria, en algunos contextos, el profesional de enfermería tarda en incorporar a su práctica el uso de evidencia, debido al acceso inadecuado a revistas de investigación, falta de conocimiento sobre cómo implementarlas, entre otras. Aunado a que los metanálisis y meta-síntesis realizados en enfermería han sido limitados en comparación con otras disciplinas. Además, se ha generado evidencia a través de la investigación en enfermería, pero se necesitan estudios adicionales que se centren en determinar la efectividad de las intervenciones de enfermería en los resultados de cuidado, a través de estudios experimentales y cuasiexperimentales para generar pruebas robustas para la práctica. Lo anterior es una oportunidad para trabajar conjuntamente las Instituciones de Educación Superior y las Instituciones de Salud en tomar un rol activo para garantizar que el cuidado brindado a las personas y sus familias se base en la mejor evidencia disponible.

\author{
Dra. Alicia Álvarez Aguirre \\ Doctora en Ciencias de Enfermería \\ Departamento de Enfermería Clínica \\ Universidad de Guanajuato \\ alicia.alvarez@ugto.mx
}

División de Ciencias de la Salud e Ingenierías Campus Celaya-Salvatierra

\section{REFERENCIAS BIBLIOGRÁFICAS}

1.Falconí C, Brito CJ, Verkovitch I. Integración de la enseñanza de la práctica de enfermería basada en la evidencia científica. Aquichan. 2015 ; 15 (4): 541 - 553. doi: 10.5294/aqui.2015.15.4.9.

2.Grove SK, Burns N, Gray JR. Understanding nursing research: Building an evidence-based practice. 5th ed. Salt Lake City: Elsevier Health Sciences, $2014:$ 1-30.

3.Volpato M, Caliri MH, Galdino MJ, Martins J. Percepción de estudiantes de enfermería sobre el aprendizaje basado en evidencias. Investig Enferm Imagen Desarr. 2018; 20(1):1-10. doi: https://doi.org/10. 11144/Javeriana.ie20-1.peea.

4.Cortez S, Dietrich M, Wells N. Measuring clinical decision support influence on evidence-based nursing practice. ONF. 2016, 43(4), 170-177. doi: https://doi. org/10.1188/16.ONF.E170-E177

5. Secretaría de Salud. Catálogo Maestro Guías de práctica clínica. CENETEC. 2018. http://cenetec-difusion.com/gpc-sns/?cat=157 